\title{
A indexação de assunto em documentos arquivísticos: convergências e divergências em artigos internacionais
}

\author{
Graziela Martins de Medeiros \\ Doutoranda; Universidade Federal de Santa Catarina, Florianópolis, SC, Brasil; \\ graziela.m@ufsc.br \\ Marisa Bräscher \\ Doutora; Universidade Federal de Santa Catarina, Florianópolis, SC, Brasil; \\ marisa.brascher@gmail.com \\ William Barbosa Vianna \\ Doutor; Universidade Federal de Santa Catarina, Florianópolis, SC, Brasil; \\ wpwilliam@hotmail.com
}

\begin{abstract}
Resumo: Este artigo tem como objetivo analisar as convergências e divergências apresentadas em artigos científicos internacionais que tratam sobre a indexação de assunto em documentos arquivísticos, para o avanço da temática no campo da Arquivologia e Ciência da Informação. A pesquisa é exploratóriodescritiva e qualitativa. Coletaram-se artigos de duas bases de dados: Library and Information Science Abstracts e Library, Information Science \& Technologycal Abstracts. Após análise preliminar, foram selecionados 20 artigos, que cobrem o período de 1965 a 2013. Os resultados mostram que as definições apresentadas para a indexação em Arquivologia, grosso modo, se assemelham ao entendimento de indexação no contexto da Biblioteconomia e Ciência da Informação, com a necessidade de se considerar o contexto específico e os princípios arquivísticos no processo de representação e o desenvolvimento de diretrizes para o controle vocabular.
\end{abstract}

Palavras-chave: Indexação de assunto. Representação da informação. Documentos arquivísticos.

\section{Introdução}

A representação da informação é estudada na área de Ciência da Informação (CI) visando essencialmente otimizar o processo de recuperação da informação e possibilitar seu acesso e uso. O tema também se reveste de interesse para a Arquivologia, área que se encontra em desenvolvimento teórico, especialmente no que tange às questões de representação. 
No campo da Arquivologia, a representação da informação vem se desenvolvendo em diálogo com a Biblioteconomia e a Ciência da Informação e ocorre por meio das funções arquivísticas de classificação e descrição. A primeira dessas funções pode ser entendida como o processo de organização dos documentos de arquivo, que recupera o contexto de produção e os organiza de acordo com a estrutura e as funções desempenhadas pelo organismo produtor. Já a segunda, conforme indica Rodrigues (2003), busca descrever e possibilitar o acesso às informações arquivísticas por meio da criação de instrumentos de pesquisa.

A descrição ocorre por meio da aplicação de normas, sendo o padrão internacional a Norma Geral Internacional de Descrição Arquivística $\operatorname{ISAD}(\mathrm{G})$ e o brasileiro a Norma Brasileira de Descrição Arquivística (NOBRADE), elaborada em 2006, com o objetivo de adaptar as normas internacionais à realidade brasileira.

Ambas apresentam regras para o preenchimento dos campos de descrição, porém a NOBRADE acrescenta uma nova área destinada à representação do assunto. Apesar de ter avançado com a criação deste campo, não consta na norma como fazer esta representação, bem como sua definição.

Além disso, autores da literatura da área de Arquivologia, tais como Ribeiro (1996a, 2013), Campos (2006) e Barros (2015), destacam a existência da indexação de assunto na representação de documentos de arquivo, porém indicam a necessidade de aprofundamento teórico sobre a temática.

Ribeiro (2013, p. 536) relata que depois de duas décadas o tema ainda não aparece aprofundado na literatura da área arquivística internacional:

[...] é legítimo que nos questionemos sobre o modo como evoluiu o tratamento da informação por assunto nos arquivos e que nos interroguemos sobre como se tem processado a integração dos arquivos na sociedade digital e em rede em que nos movimentamos. Não se conhecem estudos de síntese que analisem esta problemática [...].

Diante dessa lacuna teórica, este artigo tem como objetivo analisar artigos científicos internacionais que tratam sobre a indexação de assunto em documentos arquivísticos, buscando identificar as possíveis convergências e divergências apresentadas entre os autores. 
Acredita-se que as convergências podem sustentar um aporte teórico e metodológico organizado, coerente e válido para o processo de indexação em Arquivologia, contribuindo significativamente para o desenvolvimento do tema na área de Arquivologia e Ciência da Informação. O estudo contribui, ainda, para o diálogo entre as referidas áreas e para a abertura de novos campos de estudo.

Analisaram-se 20 artigos científicos disponibilizados nas bases de dados Library and Information Science Abstracts (LISA) e Library, Information Science \& Technological Abstracts (LISTA) quanto aos seguintes elementos, os quais foram especificados na metodologia: foco, denominação utilizada, definição, função arquivística, relação com grupo de trabalho específico sobre o tema.

Esclarece-se que foi adotada neste artigo a denominação "indexação de assunto" para se referir ao processo de representação de assunto realizado em documentos de arquivo, por meio da análise de seu conteúdo, podendo haver na literatura da área outras denominações e entendimentos, sendo o intuito deste artigo identificar as possíveis diferenciações.

\section{Epistemologia da Ciência da Informação}

O objetivo desta seção é mostrar o entendimento da área de Ciência da Informação, a partir de um breve panorama de seu surgimento e desenvolvimento, buscando-se relacioná-la com a área de Arquivologia. Cabe destacar inicialmente o conceito de Epistemologia: “[...] no sentido bem amplo do termo, podemos considerar o estudo metódico e reflexivo do saber, de sua organização, de sua formação, de seu desenvolvimento, de seu funcionamento e de seus produtos intelectuais." (JAPIASSU, 1977, p. 16).

Quanto à Ciência da Informação, as divergências epistemológicas já se iniciam quando se trata do seu surgimento. Para alguns autores, está área surge a partir das discussões ocorridas nas reuniões do Georgia Institute of Technology, realizadas nos Estados Unidos da América (EUA) em 1961/1962 (PINHEIRO, 1998, 2005; SARACEVIC, 1996; RABELLO, 2012). Numa dessas reuniões, 
surgiu o conceito elaborado por Borko (1968), segundo o qual "Ciência da Informação é a disciplina que investiga as propriedades e o comportamento da informação, as forças que governam o fluxo da informação e os meios de processamento da informação para acessibilidade e usabilidade ótimas." (BORKO, 1968, p. 2-3, tradução nossa).

Shera e Cleveland (1977) foram pioneiros ao escrever sobre o surgimento da Ciência da Informação, formulando um texto acerca de sua história e fundamentos. Na visão desses autores, a Ciência da Informação nasce a partir da área de Documentação e devido ao acelerado desenvolvimento tecnológico observado na década de 1960.

Ao tratarem sobre as perspectivas norte-americana e europeia, Shera e Cleveland (1977) afirmam que segundo esta última, a documentação surge com a iniciativa dos advogados belgas Paul Otlet e Henri La Fontaine, que tinham como objetivo desenvolver o Controle Bibliográfico Universal (CBU). Rabello (2012) complementa que a divergência entre os bibliotecários tradicionais e os que trabalhavam com bibliografias especializadas provocou, na França, uma distinção entre esses dois grupos de profissionais, distinguindo-se o segundo grupo como documentalistas, fato que também contribuiu para o surgimento da Ciência da Informação.

Nessa perspectiva, nosso objetivo não é optar por um ou outro entendimento ou corrente a respeito do surgimento da área. $\mathrm{O}$ intuito é esclarecer as divergências existentes, as quais podem repercutir no desenvolvimento da área e, logo, na visão de informação adotada.

Para tanto, a contribuição de Saracevic (1996, p. 47) é relevante, ao afirmar que:

Ciência da informação é um campo dedicado às questões científicas e à prática profissional voltadas para os problemas da efetiva comunicação do conhecimento e de seus registros entre os seres humanos, no contexto social, institucional ou individual do uso e das necessidades de informação. No tratamento destas questões são consideradas de particular interesse as vantagens das modernas tecnologias informacionais.

De acordo com a posição epistemológica do autor supramencionado, o problema fundamental da Ciência da Informação é a Recuperação da 
Informação, que é fundante e responsável pelo desenvolvimento da referida área. Além disso, Saracevic (1996) afirma que a CI possui uma natureza mutante, com três características: natureza interdisciplinar, está ligada à tecnologia da informação e participa, junto com outras disciplinas, da evolução da sociedade da informação. A expressão "natureza mutante", tratada pelo autor, deve-se ao fato da informação se modificar conforme transformações sociais, econômicas, culturais e científicas. Assim, esse fenômeno é complexo e não deve ser estudado de forma isolada.

Já a interdisciplinaridade diz respeito às disciplinas que se relacionam com a área de CI, que para Saracevic (1996) são: a Biblioteconomia, a Ciência da Computação, as Ciências Cognitivas e a Comunicação. Nesse sentido, concorda-se com Pinheiro (1998), quando afirma que Saracevic (1996) é mais restritivo que outros autores, como o próprio Borko, pois indica poucas áreas de interdisciplinaridade da Ciência da Informação. Porém, as áreas são condizentes com sua posição epistemológica, uma vez que são necessárias para o desenvolvimento da Recuperação da Informação.

Como os conceitos apresentados por Borko (1968) e Saracevic (1996) são convergentes e complementares, considera-se que os dois apresentam a ideia de Ciência da Informação adotada neste artigo, como sendo uma ciência que tem como objeto de estudo a informação, de caráter interdisciplinar por natureza, participa da sociedade da informação e é uma ciência social.

Este artigo está pautado em pontos de intersecção entre duas áreas de estudo: a Arquivologia e a Ciência da Informação, que são tratadas por meio de sua relação interdisciplinar. Silva (2013) destaca que entre as intercorrências interdisciplinares da CI existem sete tendências. Dentre elas, uma se refere à interdisciplinaridade envolvendo a Biblioteconomia e extensivamente a Arquivologia e a Museologia. 


\section{Arquivologia: origens e objeto de estudo}

As áreas que envolvem este artigo são basicamente a Ciência da Informação e a Arquivologia, sobre a qual se discorre, tendo como ponto de partida o seu surgimento, como destaca Araújo (2013, p. 52):

Aquilo que hoje é conhecido por Arquivologia tem origem no período do Renascimento, a partir do século XV, quando ressurgiu o interesse pela produção humana, pelo estudo de sua história e sua evolução política e econômica. Salientou-se o interesse pela salvaguarda e preservação dos registros das atividades humanas nas mais variadas esferas.

Araújo (2014) complementa que proliferaram, entre os séculos XVI e XVII, tratados e manuais voltados para regras e procedimentos nas instituições responsáveis pela guarda das obras. Assim, um dos primeiros elementos da disciplina Arquivística pode ser considerado o surgimento da obra De re diplomática, publicada em 1681 por Jean Mabbilon. Embora essa não seja uma obra arquivística, inspirou autores da área por meio de seus fundamentos, que serviram de base para o tratamento dos documentos arquivísticos.

Fonseca (2005) destaca também como marco inaugural do que se poderia chamar de disciplina arquivística o Manual dos Arquivistas Holandeses, de S. Muller, J. A. Feith e R. Fruin, criado dois séculos mais tarde, em 1898. Lopes (2009) esclarece que este manual inaugurou o pensamento arquivístico tradicional por suas regras e métodos de tratamento dos arquivos definitivos.

Buscando-se elementos mais significativos para o entendimento da área, cabe enfatizar que a Arquivologia apresenta três correntes de pensamento, de acordo com Lopes (2009): Arquivologia Clássica, Records Management e Arquivologia integrada. A primeira é europeia e desenvolveu-se em países como França, Itália e Espanha. Seu foco são os arquivos permanentes e, logo, de valor secundário. Recusa-se a questionar a origem, isto é, a criação, a utilização administrativa, técnica e jurídica dos arquivos.

A segunda, também conhecida como estadunidense, consiste em um conjunto de regras práticas que não possuem fundamentos científicos rigorosos, dando margem à improvisação. Preocupa-se com os arquivos correntes e intermediários, ou seja, com documentos de valor primário (LOPES, 2009). 
A última corrente (Integrada) é proveniente do Canadá, da região do Quebec e se preocupa com o ciclo completo de vida dos documentos. É a única a propor a transformação da arquivística em uma disciplina científica, aberta à pesquisa, à redefinição de conceitos e de metodologias, permitindo se autoquestionar (LOPES, 2009).

Assim, pode-se afirmar que a corrente que mais se aproxima da proposta deste artigo é a Integrada, por representar a ideia de Arquivologia pós-custodial e pós-moderna, numa perspectiva contemporânea da Arquivologia, conforme destaca Araújo (2014). Porém, não se pode dizer que autores clássicos da Arquivologia não podem contribuir para a pesquisa, visto que há uma fragmentação do pensamento arquivístico internacional, conforme destacou Lopes (2009). Assim, não há uma unidade entre os autores, tampouco aplicação dos pressupostos de cada corrente ou teoria separadamente na prática arquivística, especialmente no caso do Brasil, cenário onde se desenvolve esta pesquisa.

Outra questão a ser enfatizada é o objeto de estudo da Arquivologia: a informação ou o documento arquivístico. Recentemente, Schmidt (2013) apresentou um trabalho fruto de sua tese de doutorado, que discute o objeto científico da Arquivologia. Em sua concepação:

[...] há duas 'possibilidades informacionais' no campo dos arquivos. Uma, diz respeito ao que está 'dentro' do documento, o seu conteúdo. A outra, é aquela que está 'fora' do documento, isto é, quem o produziu, por que, para quê, quando, onde e como, além de seu trâmite e seus vínculos. (SCHMIDT, 2013, p. 17).

Em outras palavras, as informações que estão fora dizem respeito ao contexto de produção dos documentos e, logo, sua relação com as funções e a estrutura da instituição produtora/acumuladora, enquanto as informações que estão dentro se referem ao seu conteúdo informativo.

Assim, pode-se dizer que o documento arquivístico é um artefato humano com pressupostos e características específicas, o qual representa o produto da atividade do sujeito acumulador, conforme destaca Sousa (2008). Dessa maneira, os documentos são produzidos e organizados levando-se em 
conta princípios arquivísticos, destacados por Bellotto (2002), a saber: proveniência, organicidade, unicidade e indivisibilidade. Esses princípios estão relacionados, ou seja, os documentos arquivísticos basicamente não devem ser misturados a documentos de proveniências distintas; são criados a partir de funções e atividades da instituição (conjuntos orgânicos), são únicos no contexto em que foram produzidos e são conjuntos indivisíveis.

Como não há consenso na área de Arquivologia quanto ao uso dos termos "documento de arquivo" ou "informação arquivística", conforme destacou Schmidt (2013), e, de acordo com as características do objeto de estudo desta área, adotou-se neste artigo a expressão "documento arquivístico" para se referir à informação arquivística registrada, em qualquer suporte.

\section{Representação da informação}

De acordo com a proposta apresentada por Bräscher e Café (2010), o processo de organização da informação (OI) resulta na representação da informação (RI). Esta última pode ser compreendida como o conjunto de atributos que representam determinado objeto informacional e que é obtida pelos processos de descrição física e de conteúdo. Já a representação do conhecimento (RC), oriunda do processo de organização do conhecimento (OC), se constitui numa estrutura conceitual que representa modelos de mundo, visando a construção de Sistemas de Organização do Conhecimento (SOC).

Alguns autores não diferenciam a $\mathrm{OC}$ da OI, como parece ser o caso de Esteban Navarro (1995, p. 66, tradução nossa), que ao tratar a respeito da organização e representação do conhecimento no contexto da Arquivologia afirma que:

Por representação e organização do conhecimento entendemos a disciplina dedicada à investigação e fundamentos científicos e desenvolvimento das técnicas de planejamento, construção, uso, gestão e avaliação das habilidades e ferramentas empregadas nos sistemas documentais para o armazenamento, tratamento e recuperação dos documentos criados pela humanidade para testemunhar seus atos $[\ldots]$. 
Percebe-se que o conceito apresentado pelo autor para a "organização e representação do conhecimento" é abrangente e envolve todo o processo de gestão documental, desde a fase de produção até a recuperação.

Considerando a característica orgânico-funcional dos documentos de arquivo, ressalta-se que esses devem ser organizados, representados e recuperados em conjuntos documentais, considerando os princípios arquivísticos, como a proveniência e a ordem original, destacados por Rousseau e Couture (1998). Quanto a estes princípios, juntamente com outros tratados na seção anterior, Esteban Navarro (1995) assevera que cada agrupamento consiste em um todo que não pode ser separado, porque as partes que lhe compõem só fazem sentido enquanto permanecem unidas no conjunto no qual nasceram.

Vale destacar que a representação da informação tem como objetivo a recuperação e o acesso à informação, tanto na Ciência da Informação como na Arquivologia. Nesse sentido, as etapas de entrada, processamento e saída podem ser utilizadas no tratamento documental nas duas áreas, visto que são comuns a qualquer processo de recuperação da informação. Segundo Smit (2013), na área de Arquivologia, a descrição e classificação produzem a "entrada" do sistema, enquanto o acesso, por sua vez, constitui a "saída".

Verifica-se que na saída do sistema pode ocorrer o processo de indexação, visando otimizar a recuperação e acesso à informação. Como instrumento de armazenagem e recuperação da informação, a indexação era utilizada na biblioteca de Alexandria e na classificação de Calímaco. Mais tarde, fora utilizada na organização de inventários de manuscritos medievais (CAVALCANTI, 1978).

No domínio da Ciência da Informação, a indexação pode ser definida como o "[...] ato de identificar e descrever o conteúdo de um documento em termos representativos dos seus assuntos e que constituem uma linguagem de indexação.” (ASSOCIAÇÃO BRASILEIRA DE NORMAS TÉCNICAS, 1992). Esse processo envolve duas etapas: a extração dos conceitos que possam representar o assunto de um documento e a tradução desse conceito para uma linguagem de indexação (DIAS; NAVES, 2013). 
Nesse artigo concorda-se com proposta conceitual de Bräscher e Café (2010), principalmente quando afirmam que indexação está relacionada à "descrição de conteúdos", que compreende a elaboração de resumos, a classificação e a indexação.

As questões relativas à indexação de assunto na área de Arquivologia encontram-se discutidas na seção de resultados deste artigo e, por este motivo, não são aqui aprofundadas.

\section{Procedimentos metodológicos}

A pesquisa é de caráter exploratório-descritivo. De acordo com Gil (2008, p. 27), as pesquisas exploratórias:

[...] são desenvolvidas com o objetivo de proporcionar visão geral, do tipo aproximativo, acerca de determinado fato. Este tipo de pesquisa é realizado especialmente quando o tema escolhido é pouco explorado e torna-se difícil sobre ele formular hipóteses precisas e operacionalizáveis.

Já as descritivas “[...] têm como objetivo primordial a descrição das características de determinada população ou fenômeno ou o estabelecimento de relações entre variáveis.” (GIL, 2008, p. 28).

Utiliza-se como procedimento metodológico a pesquisa bibliográfica, com base em artigos das bases de dados: Library and Information Science Abstracts (LISA) e Library, Information Science \& Technologycal Abstracts (LISTA). A seleção levou em consideração bases de dados internacionais representativas da área de Ciência da Informação, sendo a primeira referencial e de resumo e a segunda, por sua vez, de texto completo.

Para a coleta nas bases de dados internacionais utilizou-se o termo Indexing combinado com Archives ou Archival Science, nos campos de busca avançada, selecionando-se somente por "publicações científicas revisadas por pares". Esclarece-se que não se utilizou o termo Subject indexing para as buscas, pois o mesmo não retornou resultados.

Foram considerados somente artigos científicos, desconsiderando-se aqueles cujo texto completo não estava disponível e, ainda, anais de eventos e outros tipos de publicações. Além disso, não se estabeleceu um recorte 

convergências e divergências em artigos internacionais Graziela Martins de Medeiros, Marisa Bräsher e William Barbosa Vianna

temporal, tendo em vista verificar desde quando o tema aparece na literatura da área. No total foram selecionados 20 artigos, conforme o Quadro 1.

Quadro 1 - Artigos analisados

\begin{tabular}{|c|c|c|c|c|}
\hline $\mathbf{N}^{\mathbf{0}}$ & Autores & Título & Dados da publicação & Ano \\
\hline 1 & Johnson, L C. & Archive indexing & $\begin{array}{l}\text { Indexer, v. 4, n. 4, } \\
\text { Autumn, p.105-108. 4p. }\end{array}$ & 1965 \\
\hline 2 & Storey, R. A. & Indexing archives & $\begin{array}{l}\text { Indexer, v. 5, n. 4, } \\
\text { Autumn, p. 159-168. }\end{array}$ & 1967 \\
\hline 3 & Lytle, R. H. & $\begin{array}{c}\text { Intellectual Access to } \\
\text { Archives: II. Report of an } \\
\text { Experiment Comparing } \\
\text { Provenance and Content } \\
\text { Indexing Methods of Subject } \\
\text { Retrieval }\end{array}$ & $\begin{array}{c}\text { The American Archivist, } \\
\text { v. } 43, \text { n. } 2 \text {, Spring. }\end{array}$ & 1980 \\
\hline 4 & Dryden, J. E. & $\begin{array}{l}\text { Subject Headings: the } \\
\text { PAASH Experience }\end{array}$ & $\begin{array}{c}\text { Archivaria: The Journal } \\
\text { of The Association of } \\
\text { Canadian Archivsts, v. 24, } \\
\text { Summer. }\end{array}$ & 1987 \\
\hline 5 & McNitt, W. H. & $\begin{array}{c}\text { Development of the } \\
\text { PRESNET subject descriptor } \\
\text { thesaurus }\end{array}$ & $\begin{array}{c}\text { American Archivist, v. } \\
\text { 52, n. } 3 .\end{array}$ & 1989 \\
\hline 6 & Ostroff, $\mathbf{H}$. & $\begin{array}{c}\text { Subject Access to Archival } \\
\text { and Manuscript Material }\end{array}$ & $\begin{array}{c}\text { American Archivist, } \mathrm{v} . \\
53, \mathrm{n} .1\end{array}$ & 1990 \\
\hline 7 & $\begin{array}{l}\text { Smiraglia, } \\
\text { R. P. }\end{array}$ & $\begin{array}{c}\text { Subject Access to Archival } \\
\text { Materials Using LCSH }\end{array}$ & $\begin{array}{c}\text { Catalogingand } \\
\text { Classification Quarterly, } \\
\text { v. } 11, \text { n. } 3-4 .\end{array}$ & 1990 \\
\hline 8 & Dooley, J. M. & Subject indexing in context & $\begin{array}{l}\text { American Archivist, v. } \\
\text { 55, n.2, Spring, p.344- } \\
354 \text {. }\end{array}$ & 1992 \\
\hline 9 & MacNeil, $\mathbf{H}$. & $\begin{array}{c}\text { Subject access to archival } \\
\text { fonds: balancing provenance } \\
\text { and pertinence }\end{array}$ & $\begin{array}{l}\text { Fontes Artis Musicae, v. } \\
\text { 43, n. } 3 \text {, July-September, } \\
\text { p. } 242-258 .\end{array}$ & 1996 \\
\hline 10 & Ribeiro, F. & $\begin{array}{c}\text { Subject indexing and } \\
\text { authority control in } \\
\text { archives: the need for } \\
\text { subject indexing in archives } \\
\text { and for an indexing policy } \\
\text { using controlled language }\end{array}$ & $\begin{array}{c}\text { Journal of the Society of } \\
\text { Archivists, v. } 17, \text { n. } 1, \\
\text { April. }\end{array}$ & 1996 \\
\hline 11 & $\begin{array}{l}\text { Gagnon- } \\
\text { Arguin, L. }\end{array}$ & $\begin{array}{l}\text { Documentary analysis } 2: \\
\text { Current problems relating to } \\
\text { documentary analysis. }\end{array}$ & $\begin{array}{c}\text { Archives, v. } 28, \text { n. 3-4, p. } \\
\text { 23-41. }\end{array}$ & $\begin{array}{c}1996 \\
- \\
1997 \\
\end{array}$ \\
\hline 12 & Hodgson, $\mathbf{N}$. & $\begin{array}{c}\text { Analysedocumentaire III: } \\
\text { Thésaurusetfichier } \\
\text { d'autorité à l'Université } \\
\text { Concordia }\end{array}$ & Archives, v.28, n.3. & $\begin{array}{c}1996 \\
- \\
1997\end{array}$ \\
\hline 13 & Hudon, M. & $\begin{array}{c}\text { Indexing and documentary } \\
\text { languages in archival } \\
\text { environments in the era of } \\
\text { new information } \\
\text { technologies }\end{array}$ & $\begin{array}{c}\text { Archives, v. 29, n. 1, p. } \\
75-98 .\end{array}$ & $\begin{array}{c}1997 \\
- \\
1998\end{array}$ \\
\hline 14 & Elings, M. W. & $\begin{array}{l}\text { Pictoral Archives and EAD: } \\
\text { Indexing Collections for }\end{array}$ & $\begin{array}{l}\text { Art Documentation, } \mathrm{v} . \\
19, \text { n. } 2, \text { p. } 10-14 .\end{array}$ & 2000 \\
\hline
\end{tabular}




\begin{tabular}{|c|c|c|c|c|}
\hline & & Online Access & & \\
\hline 15 & Garrod, P. & $\begin{array}{c}\text { Use of the UNESCO } \\
\text { Thesaurus for archival } \\
\text { subject indexing at UK } \\
\text { NDAD }\end{array}$ & $\begin{array}{c}\text { Journal of the Society of } \\
\text { Archivists } \\
\text { v. } 21, \text { n. } 1, \text { p. } 37-54 .\end{array}$ & 2000 \\
\hline 16 & Lévesque, M. & $\begin{array}{c}\text { L'indexation: luxe ou } \\
\text { necessite? }\end{array}$ & $\begin{array}{l}\text { Archives, v. } 33 \text {, n.1, } \\
\text { 2001, p. } 17-45\end{array}$ & $\begin{array}{c}2001 \\
- \\
2002\end{array}$ \\
\hline 17 & Hicks, S. & Indexing archives for access & $\begin{array}{c}\text { The Indexer, v. 24, n. 4, } \\
\text { October. }\end{array}$ & 2005 \\
\hline 18 & $\begin{array}{l}\text { Schenkolewski } \\
\text {-Kroll, S.; } \\
\text { Tractinsky, A. }\end{array}$ & $\begin{array}{l}\text { Archival Description, } \\
\text { Information Retrieval, and } \\
\text { the Construction of Thesauri } \\
\text { in Israeli Archives }\end{array}$ & $\begin{array}{c}\text { Archival Science, v. 6, n. } \\
\text { 1, p. 69-107. }\end{array}$ & 2006 \\
\hline 19 & Fenton, $C$. & $\begin{array}{c}\text { Use of Controlled } \\
\text { Vocabulary and Thesauri in } \\
\text { UK Online Finding Aids }\end{array}$ & $\begin{array}{c}\text { Journal of the Society of } \\
\text { Archivists } \\
\text { v. } 31, \text { n. } 2, \text { p. } 187-205 .\end{array}$ & 2010 \\
\hline 20 & Guitard, L. A. & $\begin{array}{c}\text { Indexation par sujet en } \\
\text { archivistique et en } \\
\text { bibliothéconomie: du pareil } \\
\text { au meme }\end{array}$ & $\begin{array}{c}\text { Documentation et } \\
\text { bibliothèques, v. } 59, \mathrm{n} . \\
\text { 4, p. 201-212. }\end{array}$ & 2013 \\
\hline
\end{tabular}

Fonte: Elaborado pelos autores.

A análise dos artigos orienta-se pelos seguintes elementos:

a) natureza do estudo - teórico, prático ou teórico-prático;

b) foco - aspecto central do texto, sintetizado com base na leitura do título, resumo, palavras-chave e texto completo;

c) denominação - termo(s) adotado(s) para se referir à indexação de assunto;

d) definição apresentada para "indexação de assunto", em documentos arquivísticos;

e) função - relação da indexação de assunto com as funções arquivísticas;

f) relação do texto publicado com o trabalho de algum grupo de trabalho ou estudo específico.

\section{Indexação de assunto em documentos arquivísticos: resultados e discussão}

Quanto aos anos de publicação, verifica-se que no período de 1965 a 2013 foram identificados 20 artigos sobre indexação em arquivos nas bases de dados pesquisadas. Entre 1965 e 1989 foram publicados apenas cinco. A maior 
incidência de publicações foi de 1990 a 1999, período em que recuperam-se oito publicações, ao passo que de 2000 a 2013 localizou-se apenas sete.

Os 20 artigos distribuem-se em 10 periódicos distintos. Desses, apenas quatro apresentaram mais de um artigo sobre o tema: American Archivist (4), Archives (4), The Indexer (3) e Journal of the Society of Archivists (3). As demais publicações apresentaram apenas um artigo cada: Archivaria, Art Documentation, Fontes Artis Musicae, Archival Science, Cataloging and Classification Quarterly, Documentation et bibliothèques. O Quadro 2 apresenta a síntese da análise efetuada. Não se inseriu o foco, pois está apresentado na discussão subsequente ao quadro.

Quadro 2 - Síntese da análise efetuada.

\begin{tabular}{|c|c|c|c|c|c|c|}
\hline $\mathbf{N}^{\mathbf{0}}$ & Autor (ano) & Natureza & Denominação & Definição & Função & Grupo \\
\hline 1 & $\begin{array}{c}\text { Johnson } \\
(\mathbf{1 9 6 5 )} \\
\end{array}$ & Prático & Indexing & --- & --- & --- \\
\hline 2 & $\begin{array}{l}\text { Storey } \\
(1967)\end{array}$ & Prático & Indexing & --- & --- & --- \\
\hline 3 & Lytle (1980) & Prático & $\begin{array}{c}\text { Content } \\
\text { indexing; } \\
\text { Subject } \\
\text { retrieval }\end{array}$ & Sim & --- & --- \\
\hline 4 & $\begin{array}{c}\text { Dryden } \\
(1987)\end{array}$ & Prático & $\begin{array}{l}\text { Subject } \\
\text { Headings; } \\
\text { Subject } \\
\text { Access. }\end{array}$ & --- & --- & --- \\
\hline 5 & $\begin{array}{l}\text { McNitt } \\
\text { (1989) }\end{array}$ & Prático & $\begin{array}{c}\text { Subject } \\
\text { access; } \\
\text { subject index } \\
\text { system }\end{array}$ & --- & $\begin{array}{l}\text { Classifiação; } \\
\text { Descrição }\end{array}$ & --- \\
\hline 6 & $\begin{array}{c}\text { Ostroff, } \mathrm{H} . \\
\text { (1990) }\end{array}$ & Prático & Subject access & --- & Descrição & $\begin{array}{c}\text { Bureau of } \\
\text { Canadian } \\
\text { Archivists } \\
\text { (BCA) }\end{array}$ \\
\hline 7 & $\begin{array}{c}\text { Smiraglia } \\
(1990)\end{array}$ & $\begin{array}{l}\text { Teórico- } \\
\text { prático }\end{array}$ & $\begin{array}{c}\text { Subject access } \\
\text { points; } \\
\text { Subject } \\
\text { analysis }\end{array}$ & --- & --- & --- \\
\hline 8 & $\begin{array}{l}\text { Dooley } \\
(1992)\end{array}$ & $\begin{array}{l}\text { Teórico- } \\
\text { prático }\end{array}$ & $\begin{array}{c}\text { Subject } \\
\text { indexing }\end{array}$ & --- & Descrição & $\mathrm{BCA}$ \\
\hline 9 & $\begin{array}{c}\text { MacNeil } \\
\text { (1996) }\end{array}$ & Teórico & $\begin{array}{c}\text { Subject } \\
\text { indexing; } \\
\text { Subject access }\end{array}$ & Sim & Descrição & $\mathrm{BCA}$ \\
\hline 10 & $\begin{array}{c}\text { Ribeiro } \\
\text { (1996) }\end{array}$ & $\begin{array}{l}\text { Teórico- } \\
\text { prático }\end{array}$ & $\begin{array}{c}\text { Subject } \\
\text { indexing }\end{array}$ & --- & Descrição & --- \\
\hline 11 & $\begin{array}{c}\text { Gagnon- } \\
\text { Arguin } \\
(1996-1997)\end{array}$ & Teórico & $\begin{array}{l}\text { l'indexation } \\
\text { par sujet; } \\
\text { L'accès par } \\
\text { sujet }\end{array}$ & Sim & Descrição & \\
\hline 12 & Hodgson & Teórico- & l'indexation & --- & --- & BCA \\
\hline
\end{tabular}




\begin{tabular}{|c|c|c|c|c|c|c|}
\hline & (1996-1997) & prático & par sujet & & & \\
\hline 13 & $\begin{array}{c}\text { Hudon } \\
(1997-1998)\end{array}$ & $\begin{array}{l}\text { Teórico- } \\
\text { prático }\end{array}$ & $\begin{array}{l}\text { l'indexation } \\
\text { par sujet; } \\
\text { L'indexation } \\
\text { thématique }\end{array}$ & Sim & Descrição & BCA \\
\hline 14 & $\begin{array}{l}\text { Elings } \\
(2000)\end{array}$ & Prático & $\begin{array}{c}\text { Subject } \\
\text { indexing; } \\
\text { Subject access }\end{array}$ & --- & Descrição & --- \\
\hline 15 & $\begin{array}{c}\text { Garrod } \\
(2000)\end{array}$ & Prático & $\begin{array}{c}\text { Subject } \\
\text { indexing }\end{array}$ & --- & Descrição & --- \\
\hline 16 & $\begin{array}{l}\text { Lévesque } \\
\text { (2001-2002) }\end{array}$ & Teórico & L'indexation & --- & $\begin{array}{c}\text { Classificaçã } \\
\text { o; } \\
\text { Descrição } \\
\end{array}$ & --- \\
\hline 17 & $\begin{array}{l}\text { Hicks } \\
\text { (2005) }\end{array}$ & $\begin{array}{l}\text { Teórico- } \\
\text { prático }\end{array}$ & $\begin{array}{l}\text { Archival } \\
\text { indexing; } \\
\text { Indexing } \\
\text { archival } \\
\text { records }\end{array}$ & --- & --- & $\begin{array}{l}\text { The } \\
\text { australian } \\
\text { and New } \\
\text { Zeland } \\
\text { Society of } \\
\text { Indexers } \\
\text { (ANZSI) }\end{array}$ \\
\hline 18 & $\begin{array}{c}\text { Schenkolew } \\
\text { ski; } \\
\text { Tractinsky } \\
(2006) \\
\end{array}$ & $\begin{array}{l}\text { Teórico- } \\
\text { Prático }\end{array}$ & $\begin{array}{l}\text { Subject } \\
\text { indexing }\end{array}$ & --- & --- & --- \\
\hline 19 & $\begin{array}{c}\text { Fenton } \\
(2010) \\
\end{array}$ & Prático & $\begin{array}{c}\text { Subject } \\
\text { indexing } \\
\end{array}$ & --- & --- & --- \\
\hline 20 & $\begin{array}{c}\text { Guitard } \\
\text { (2013) }\end{array}$ & Teórico & $\begin{array}{c}\text { l'indexation } \\
\text { parsujet }\end{array}$ & Sim & Descrição & $\mathrm{BCA}$ \\
\hline
\end{tabular}

Fonte: Elaborado pelos autores.

Dentre os textos de cunho teórico e que apresentam definições sobre os processos de indexação de assunto, acesso por assunto e análise de assunto estão: MacNeil (1996), Gagnon-Arguin (1996-1997), Hudon (1997-1998), Lévesque (2001-2002) e Guitard (2013).

Dentre esses, destaca-se o de Heather MacNeil, publicado em Vancouver, no Canadá, em 1996, como uma das relevantes contribuições teóricas a respeito da indexação arquivística. Em sua visão, a indexação por assunto nos arquivos se desenvolveu nacional e internacionalmente por meio das normas de descrição. Assim, a autora compreende a indexação como o processo de análise do assunto e do contexto documental para realizar a atividade de descrição, especificamente a descrição multinível feita por meio de normas de descrição.

A autora utiliza a expressão "descrição e indexação", pois considera que a descrição multinível gera, por sua vez, uma indexação multinível. Quanto às denominações, utiliza as expressões Subject indexing e Subject access, ou seja, 
indexação de assunto e acesso por assunto. Apresenta a seguinte definição para Subject indexing: "Indexação de assunto é uma abordagem direta para alcançar o acesso por tema com base no assunto do documento. Termos do índice de assunto indicam diretamente sobre o que trata um documento e são selecionados com base na análise do conteúdo de um documento" (MACNEIL, 1996, tradução nossa).

Vale destacar a preocupação da autora supracitada com os princípios arquivísticos, pois relaciona a indexação com a proveniência. Assim, um dos aspectos enfatizados no texto de MacNeil (1996) é que para descrever o documento arquivístico é necessário entender o significado amplo do contexto de produção em que o documento foi criado e sob o qual tem sido analisado e elaborado. A observância desse princípio é feita na descrição multinível, segundo a qual o fundo de arquivo é descrito do geral para o específico, em diferentes níveis, que representam diferentes partes do fundo (conjunto documental).

Os canadenses da região do Quebec, no contexto dos artigos integrantes deste corpus de análise, representam o grupo mais consistente de trabalho a respeito do tema. Escrevem com base em estudos do Bureau of Canadian Archivists (BCA), conforme mostra o Quadro 2. Este grupo é especializado no estudo da descrição arquivística. Em 1988, o BCA criou um Grupo de Trabalho sobre indexação em documentos arquivísticos. Os resultados foram publicados em 1992 num relatório que serve de referência para o embasamento teórico desses autores, conforme explica Hudon (1997-1998).

Assim, Gagnon-Arguin (1996-1997), Hudon (1997-1998) e Lévesque (2001-2002) são convergentes quanto à visão de representação de assunto apresentada, uma vez que relacionam esse processo à descrição e consideram o assunto como uma forma adicional de acesso.

Gagnon-Arguin (1996-1997) afirma que o tratamento de assunto em arquivos tem sido uma preocupação crescente entre os arquivistas Quebequenses desde 1986, expressa por meio de publicações sobre o tema e por iniciativas de utilização da indexação de assunto ou de cabeçalhos de assuntos relacionados às normas de descrição arquivística, como forma de melhorar o acesso aos 
documentos de arquivo. Foi desenvolvida uma norma de descrição arquivística para o Quebec na década de 1990, a qual destaca a preocupação com os usuários e suas formas de busca no processo de recuperação da informação. O referido autor acredita que o acesso por assunto ou por tema é possível por meio de guias temáticos.

Hudon (1997-1998) explicita a relação existente entre o processo de indexação de assuntos em Arquivologia e a descrição arquivística e, consequentemente, os níveis de descrição, informando que qualquer política de indexação estará necessariamente ligada à definição de políticas de descrição vigentes. Acrescenta que a organização e estruturação da informação por conteúdo é apenas um acesso adicional. O artigo indica como desafios o estabelecimento de políticas de indexação, sistemas de indexação e linguagens documentárias eficazes pelos arquivistas.

Em relação à definição, Hudon (1997-1998) afirma que em arquivos, a indexação não serve para organizar ou estruturar as coleções de conteúdo. A indexação temática serve estritamente para fornecer acesso adicional àquele já existente. Além disso, as formas de acesso raramente levam à um documento particular, mas a todos documentos já agrupados em um fundo, em uma série ou em um dossiê.

Ou seja, o objetivo da indexação na comunidade de arquivo parece ser diferente daquele existente em outras áreas. Em arquivos, a indexação é aplicada para fornecer acesso adicional por meio de pontos de acesso, conforme visão de Hudon (1997-1998).

Lévesque (2001-2002) enfatiza que os arquivistas costumam privilegiar outras formas de acesso aos documentos de arquivo que não o seu conteúdo. $\mathrm{O}$ autor evidencia que o contexto é o ponto de vista mais privilegiado na teoria da área. Porém, o arquivista deve ajudar o pesquisador ou usuário a fornecer diferentes formas de acesso para facilitar as suas pesquisas.

Em relação à denominação adotada, o autor utiliza L'indexation, e apresenta definições gerais, advindas de autores que publicam sobre indexação em um contexto mais amplo da Biblioteconomia e Ciência da Informação, como a definição destacada a seguir: 
[...] do ponto de vista do processo, a indexação é a operação que consite em descrever e caracterizar um documento usando representações dos conceitos contidos no documento, ou seja, para transcrever em linguagem documentária os conceitos depois de serem extraídos a partir da análise dos documentos. (MAUREL; CHAMPANHE, $1999^{1}$, p. 318 apud LÉVESQUE, 2001-2002, tradução nossa).

Um dos artigos mais recentes e com contribuição significativa para a área é o de Guitard (2013), publicado como fruto de sua tese de doutorado. Nessa publicação, a autora destaca que no Canadá foram criadas normas de descrição arquivística em 1992, com um grupo específico para tratar da indexação de Arquivos, o já citado BCA. Neste documento foram apresentados três aspectos inerentes à indexação: o princípio de respeito aos fundos (a unidade de tratamento), os níveis de classificação (a hierarquia) e o princípio do geral para o particular. Ou seja, ao comparar o processo de indexação realizado nas áreas de Biblioteconomia e Arquivologia, pode-se verificar que as características dos documentos biblioteconômicos e arquivísticos interferem e condicionam o processo de indexação. No caso da Arquivologia, a representação deve evidenciar as informações de contexto, já que os documentos só fazem sentido no conjunto o qual fazem parte.

Entre os textos de natureza teórico-prática, estão Smiraglia (1990), Dooley (1992), Ribeiro (1996b), Hodgson (1996-1997), Hicks (2005) e Schenkolewski-kroll e Tractinsky (2006).

Smiraglia (1990) trata sobre "pontos de acesso por assunto" e "análise por assunto" nos arquivos (Subject access points e Subject analysis). O autor não explicita a função da descrição, porém utiliza o termo "catalogação", que pode ter evoluído na área de Arquivologia para a descrição. O termo Subject access points é utilizado no texto como sinônimo de Subject headings (cabeçalhos de assunto), que servem para identificar o assunto de determinados acervos arquivísticos, série, subsérie, ou itens, e para facilitar a recuperação tópica direta aos documentos.

Ribeiro (1996b), em artigo resultante de sua dissertação de mestrado sobre o tema da indexação arquivística, defendida na Universidade do Porto, Portugal, apresenta estudo empírico que compara a indexação livre e controlada 
no contexto dos arquivos. Em sua visão, os dois processos fundamentais de organização de documentos em arquivos são a descrição e o arranjo. O processo de indexação de assunto, por sua vez está relacionado à descrição e se refere à atribuição e à seleção de pontos de acesso para os documentos. Destaca que:

[...] o fator mais importante na determinação da política de indexação de assunto é a quantidade de detalhes apropriados para a descrição do conjunto de documentos envolvidos, e estes por sua vez, dependem do nível de descrição a ser utilizado. (RIBEIRO, 1996b, p. 28, tradução nossa).

Com base nessa afirmação, evidencia-se a relação que a autora faz entre a descrição e o processo de indexação de assunto.

Verifica-se que a visão apresentada por Ribeiro (1996b) é convergente com aquela apresentada por MacNeil (1996), uma vez que este último autor compreende que a indexação de assunto se refere ao estabelecimento de pontos de acesso na descrição arquivística e à elaboração de índices advindos desse processo.

Um aspecto a ser destacado é a reflexão apresentada por Ribeiro (1996b) sobre a relação entre o estabelecimento do assunto dos documentos e a classificação arquivística. Para a autora, a classificação serve para definir a estrutura dos grupos em arquivos administrativos, fazendo com que o acesso por proveniência seja possível. Porém, esse tipo de representação é apenas o complemento para buscar pelo conteúdo da informação, já que na Arquivística não se organiza (classifica) por assunto, mas sim pela estrutura do órgão e pelas funções por ele desempenhadas.

Dooley (1992) adotou a denominação Subject indexing e afirma que historicamente os arquivistas entendem o acesso por assunto nos arquivos como desnecessário. Porém, o reconhecimento da importância desse tema é crescente entre os grupos de trabalho de normas de descrição americano e canadense. No relatório publicado em 1989 pelo Working Group on Standards for Archival Description, aparece a necessidade de criação de diretrizes para a representação por assunto nos arquivos (DOOLEY, 1992). Verifica-se, nesse sentido, que a indexação de assunto, na visão do autor, se relaciona com a função de descrição 
arquivística, conforme mostra o Quadro 2. Esse autor também indica a necessidade de realização de estudos de usuário no contexto dos arquivos.

Hicks (2005) escreve a respeito dos documentos de arquivo que estão sob a custódia dos arquivos estaduais e federais australianos. Utiliza as denominações: Archival indexing e Indexing archival records e trata a indexação como um meio para conferir maior acesso aos documentos de arquivo, além de destacar os desafios que a indexação de asuntos representa para arquivos e bibliotecas. Afirma que a catalogação ou o arranjo e descrição são governados por dois princípios: proveniência (agência criadora) e ordem original (ordem na qual os documentos foram criados), porém, não há o rearranjo dos documentos por assunto ou ordem alfabética para facilitar o acesso. Ou seja, o autor se refere à criação de índices, como foi possível observar com a análise de seu artigo.

Já Schenkolewski-kroll e Tractinsky (2006) discutem a relação entre a descrição, a indexação e a recuperação da informação no contexto dos arquivos, na realidade de Israel. Os autores relacionam a indexação com a descrição arquivística. Nesse sentido, apresentam o desenvolvimento das normas de descrição em nível internacional, além de indicarem a existência de ferramentas de análise de indexação e de recuperação da informação de metadados em materiais arquivísticos.

Ao discutirem duas propostas para a construção de vocabulários controlados em Israel, os autores supracitados evidenciam a necessidade de criação de uma infraestrutura para todos os arquivos públicos daquele país.

Entre os textos de natureza prática estão Johnson (1965), Storey (1967) McNitt (1989), Dryden (1987), Garrod (2000), Lytle (1980), Ostroff (1990), Elings (2000) e Fenton (2010).

Para Dryden (1987), o assunto em documentos arquivísticos está relacionado com cabeçalhos de assunto, utilizando as denominações Subject headings e Subject access. Em sua visão, o acesso por assunto é dado por meio dos instrumentos de pesquisa. Esse autor trata do cabeçalho de assunto The Provincial Archives of Alberta Subject Headings (PAASH), desenvolvido em 1982 para dar acesso por assunto aos arquivos. 
Lytte (1980) adota as denominações Content indexing e Subject retrieval, porém sem apresentar definição a respeito. Mostra um experimento elaborado para explorar a capacidade de recuperação de assunto por proveniência e métodos de indexação de conteúdo, feito em 1978, em Baltimore, nos Estados Unidos. Elaborou testes de recuperação da informação em bases de dados, controlados por laboratório, comparando com os resultados encontrados para as buscas por "proveniência" e por "indexação de conteúdo".

Os resultados dos testes mostram que os dois métodos apresentaram baixa performance de recuperação. Uma das implicações do estudo no que tange ao tema do presente artigo, refere-se ao fato de que os sistemas de acesso por assunto nos arquivos devem ser construídos com uma expectativa razoável de que eles vão servir para uma necessidade do usuário.

Ostroff (1990) discute a utilização de listas para representar o assunto de documentos arquivísticos tendo em vista providenciar seu acesso, por meio do processo chamado por ele de "catalogação". Este termo possivelmente foi utilizado para se referir à descrição arquivística, tendo em vista que o artigo foi escrito ainda na década de 1990. Outros autores, como Smiraglia (1990) e Hicks (2005), também utilizaram esse termo em seus textos. Ostroff (1990) afirma que as listas que são utilizadas na "catalogação" apresentam problemas ao serem aplicadas aos documentos de arquivo. Assim, propõe soluções por meio uso de alguns campos do formato USMARC (formato utilizado nos EUA, baseado no Machine Readable Cataloging, o MARC).

Elings (2000) trata da especificidade dos arquivos imagéticos (ou "pictoriais", em tradução literal) e destaca o desafio de indexar este tipo de material em comparação aos documentos manuscritos, tendo em vista que os primeiros não apresentam um título previamente estabelecido, por exemplo, dificultando a identificação do seu assunto. Destaca que a "indexação de assunto", denominação por ele adotada é nos arquivos imagéticos, é utilizada nos arquivos pictoriais para facilitar seu acesso. Essa indexação é crucial tendo em vista a especificidade dos arquivos imagéticos e é feita geralmente por meio dos instrumentos de pesquisa, como guias e inventários. 
Destaca que algumas instituições arquivísticas utilizam o formato MARC para dar acesso por assunto para grandes coleções de arquivos imagéticos. Além disso, o autor enfatiza que a utilização de um formato para a codificação dos instrumentos de pesquisa, como o Encoded Archival Description (EAD), foi uma forma encontrada para as instituições arquivísticas disponibilizarem esses instrumentos online. Ao passo que esses instrumentos se tornaram mais acessíveis, a indexação de assunto passou a ser evidenciada, levantando a questão de quando e como utilizar essa indexação (ELINGS, 2000). Conclui que são necessários estudos mais aprofundados para verificar como os usuários buscam, como eles interpretam os resultados e como eles entendem os conceitos utilizados no processo de indexação. Ressalta que as normas não são claras sobre quando e como utilizar a indexação de assunto, embora esse processo seja realizado nos estudos de caso apresentados em seu texto.

Os artigos de Johnson (1965) e Storey (1967) são os mais antigos encontrados na análise e foram publicados na Revista The Indexer. O primeiro se refere ao conteúdo de uma Conferência, enquanto o segundo foca-se em metodologias, pois descreve métodos utilizados para a indexação do The National Register of Archives (NRA) e os relaciona com as necessidades dos pesquisadores. Nesse sentido, apesar de refletirem a utilização do tema em atividades práticas arquivísticas ou explicitarem a necessidade de estudo do tema desde a década de 1960, nos Estados Unidos, os referidos textos não apresentam conteúdo substancial, tais como definições ou discussão teórica.

Os outros textos de natureza prática relatam o desenvolvimento ou a utilização de tesauros no contexto arquivístico, como é o caso de McNitt (1989), Garrod (2000) e Fenton (2010).

McNitt (1989) relata a experiência de desenvolvimento de um tesauro para documentos de arquivo. Os termos adotados pelo autor foram Subject access e Subject index system. Esse segundo foi utilizado para se referir ao tesauro como um sistema de indexação de assunto, o qual mostra a relação entre os termos. Foi possível verificar que a classificação arquivística pode servir de base para a construção de linguagens de indexação para aplicação em arquivos, 
como os tesauros. Ao final do artigo, o autor mostra as lições dessa experiência. Dentre elas, a terceira consta que "[...] o tempo gasto na construção do tesauro pode ser diminuída com base em listas de assunto e esquemas de classificação existentes. " (MCNITT, 1989, tradução nossa).

Fenton (2010) trata do uso dos tesauros para a recuperação da informação de instrumentos de pesquisa online. Mostra os resultados de uma pesquisa empírica aplicada aos arquivos do Reino Unido (United Kingdom UK), por meio de questionário e foca no uso de palavras-chave de assunto nesses instrumentos.

A denominação adotada por Fenton (2010) foi Subject indexing. Com base nos resultados de sua pesquisa, o autor conclui que o número de instrumentos de pesquisa é crescente no Reino Unido, o que ocasiona a utilização de termos livres para representar os textos e, consequentemente, não contribui para a consistência da indexação.

Garrod (2000) também escreve sobre a realidade arquivística do Reino Unido e discute a utilização de tesauro para a indexação de assunto em arquivos. Verifica alguns tesauros para aplicação em arquivos, como o Tesauro do National Digital Archive of Datasets (NDAD) de UK e ao Library of Congress Subject Heandings (LCSH). O primeiro, criado de improviso, foi considerado pelo autor um índice de assunto, que utiliza a arquitetura do UNESCO Thesaurus para relacionar os termos de indexação. O segundo, por sua vez, foi rejeitado por ser mais voltado para a realidade estadunidense e ser muito amplo, não contemplando especificidades. $\mathrm{O}$ autor conclui que a segunda edição do UNESCO Thesaurus pode ser utilizada com sucesso no controle da indexação por assunto no contexto dos arquivos, informando que esse instrumento trabalha melhor no nível geral e se torna menos satisfatório nos níveis mais específicos.

\section{Considerações finais}

A pesquisa realizada permitiu verificar como a indexação de assunto é tratada nas publicações científicas de duas bases internacionais que são referência na área de Ciência da Informação: as bases LISA e LISTA. Foram recuperados 20 
artigos, que cobrem o período de 1965 a 2013, o que pode indicar uma baixa incidência de publicações sobre o tema. Vale lembrar que não foi prédeterminado um período de análise, justamente visando identificar desde quando as publicações sobre o tema aparecem na literatura internacional da área. Pesquisas dos últimos dois anos (2014 e 2015) também não foram localizadas.

No que tange aos aspectos da indexação de assunto em documentos arquivísticos, foi possível identificar sua relação com as funções arquivísticas de classificação e descrição, com ênfase na segunda. Observa-se, ainda que de maneira geral, que os autores destacam as características dos documentos de arquivo como determinantes para entender o processo de indexação nessa área, tendo em vista a complexidade e especificidade do objeto de estudo da Arquivologia.

Verifica-se que os autores canadenses identificados na análise são pioneiros quanto ao desenvolvimento do tema, visto que apontam a existência de publicações e de iniciativas institucionais em seu país a respeito do tema, especificamente na região do Quebec, desde 1986. Esses autores são convergentes quando indicam o acesso por proveniência como o principal requisito de representação dos documentos no âmbito da Arquivologia e apontam uma lacuna quanto aos estudos de representação de assunto nessa área.

É curioso observar que apenas cinco artigos pesquisados, de um total de vinte, apresentaram definição para "indexação de assunto" no contexto da Arquivologia. Pode-se afirmar que as definições apresentadas, grosso modo, se assemelham ao entendimento de indexação no contexto da Biblioteconomia e Ciência da Informação, visto que se referem ao processo de representação do assunto dos documentos para fins de recuperação. A diferença está na especificidade do documento arquivístico e, logo, na necessidade de explicitar as informações de contexto e respeitar os princípios arquivísticos em sua representação, sendo essa uma relevante lacuna a ser abordada.

Os resultados mostram que a visão mais convergente apresentada entre os autores dos artigos analisados é que a indexação em documentos arquivísticos ocorre por meio do processo de descrição, especificamente na elaboração de pontos de acesso e instrumentos de pesquisa, como índices. Os textos 
pesquisados indicam a necessidade de aprofundamento teórico sobre a temática e ressaltam a necessidade de elaboração de diretrizes de controle vocabular, a serem utilizadas na atribuição de termos de indexação de assunto em documentos arquivísticos.

Os textos da análise são oriundos de diferentes países, tais como Canadá, Estados Unidos, Austrália, Portugal e Reino Unido. Assim, é válido ressaltar que pode haver diferentes entendimentos sobre as práticas arquivísticas nos diferentes países, considerando também a existência de diferentes correntes arquivísticas, conforme destacado no referencial teórico deste artigo. Assim, os trabalhos que tratam de instrumentos de pesquisa (finding aids, em inglês) e sua relação com a representação de assunto, não necessariamente relacionam este último à descrição. Cabe essa ressalva, visto que alguns artigos da análise enfatizam esses instrumentos, porém não explicitam o processo de descrição.

Em síntese, foi possível verificar que a indexação de assunto na área de Arquivologia trata do conteúdo/assunto do documento no contexto, ou seja, como inserida no processo de descrição e como uma forma de acesso complementar. Esse aspecto, acrescido do fato de terem sido encontrados menor número de textos teórico-conceituais e da baixa incidência de textos mais recentes sobre o tema, evidencia a necessidade de realização de pesquisas que enfatizem a indexação de assunto em documentos arquivísticos, contribuindo para sua delimitação teórico-metodológica.

\section{Referências}

ARAÚJO, Carlos Alberto Ávila. Arquivologia, Biblioteconomia, Museologia e Ciência da Informação: o diálogo possível. Brasília: Briquet de Lemos, 2014.

ARAÚJO, Carlos Alberto Ávila. Epistemologia da Arquivologia: fundamentos e tendências contemporâneas. Ciência da Informação, Brasília, v. 41, n. 1, p. 5063, jan./abr. 2013.

\section{ASSOCIAÇÃO BRASILEIRA DE NORMAS TÉCNICAS. NBR 12676:}

Métodos para análise de documentos: determinação de seus assuntos e seleção de termos de indexação. Rio de Janeiro, 1992. 
BARROS, Thiago Henrique Bragato. A Indexação e a Arquivística: relações práticas e conceituais no universo teórico da representação e organização do conhecimento. In: CONGRESO ISKO ESPAÑA, 12.; CONGRESO ISKO ESPAÑA-PORTUGAL, 2., 2015, Murcia. Anais... Murcia:

Universidad de Murcia, 2015.

BELLOTO, Heloísa Liberralli. Arquivística: objetivos, princípios e rumos. São Paulo: Associação dos Arquivistas de São Paulo, 2002.

BORKO, H. Information science: what is it? American Documentation, Washington, v. 19, n. 1, p. 3-5, 1968. Disponível em: $<$ http://cdigital.uv.mx/bitstream/123456789/6699/2/Borko.pdf>. Acesso em: 23 mar. 2016.

BRÄSCHER, Marisa; CAFÉ, Ligia. Organização da informação ou organização do conhecimento? In: LARA, Marilda Lopes Ginez de; SMIT, Johanna Wilhelmina (Org.). Temas de pesquisa em Ciência da Informação no Brasil. São Paulo: USP, 2010. p. 85-102.

CAMPOS, Maria Luiza de Almeida. Indexação e descrição em arquivos: a questão da representação e recuperação de informações. Arquivo \& Administração, Brasília, v. 5, p. 17-31, 2006.

CAVALCANTI, Coedélia Robalinho. Indexação e tesauro: metodologia e técnicas. Brasília: ABDF, 1978.

DIAS, Eduardo Wense; NAVES, Madalena Martins Lopes. Análise de assunto: teoria e prática. 2. ed. rev. Brasília: Briquet de Lemos, 2013.

DOOLEY, Jackie. M. Subject indexing in context. American Archivist, Menasha, v. 55, n. 2, p. 344-354, Srping, 1992.

DRYDEN, Jean E. Subject headings: the PAASH experience. Archivaria, Ottawa, v. 24, p. 173-180, Summer 1987.

ELINGS, Mary. W. Pictoral archives and EAD: indexing collections for online access. Art Documentation, Chicago, v. 19, n. 2, p. 10-14, 2000.

ESTEBAN NAVARRO, Miguel Angel. La representación y la organización del conocimiento en los archivos. In: ENCUENTRO DE ISKO-ESPAÑA, 1., 1993, Madrid. Actas... Zaragoza: Universidad de Zaragoza: Librería General. 1995. p. 65-90.

FENTON, Candida. Use of controlled vocabulary and thesauri in UK Online finding aids. Journal of the Society of Archivists, [S.1.], v. 31, n. 2, p. 187205, 2010. 
FONSECA, Maria Odila Kahl. Arquivologia: origens e circunstâncias. In: FONSECA, Maria Odila Kahl. Arquivologia e Ciência de Informação. Rio de Janeiro: FGV, 2005. p. 55-72.

GAGNON-ARGUIN, Louise. Documentary analysis 2: current problems relating to documentary analysis. Archives, Quebec, v. 28, n. 3-4, p. 23-41, 1996-1997.

GARROD, Peter. Use of the UNESCO Thesaurus for archival subject indexing at UK NDAD. Journal of the Society of Archivists, [S.1.], v. 21, n. 1, p. 37-5, 2000.

GIL, Antônio Carlos. Métodos e técnicas de pesquisa social. 6. ed. São Paulo: Atlas, 2008.

GUITARD, Laure Amélie. Indexation par sujet en archivistique et en bibliothéconomie: du pareil au meme? Documentation et bibliothèques, Montreal, v. 59, n. 4, p. 201-212, 2013.

HICKS, Shauna. Indexing archives for access. The Indexer, London, v. 24, n. 4, Oct. 2005.

HODGSON, N. Analyse documentaire III: thésaurus et fichier d'autorité à l’Université Concordia. Archives, Quebec, v. 28, n. 3, 1996-1997.

HUDON, M. Indexing and documentary languages in archival environments in the era of new information technologies. Archives, Quebec, v. 29, n. 1, p. 75-98, 1997-1998.

JAPIASSU, Hilton. Introdução ao pensamento epistemológico. 2. ed. Rio de Janeiro: Francisco Alves, 1977.

JOHNSON, L C. Archive indexing. Indexer, London, v. 4, n. 4, p.105-108, Autumn 1965.

LÉVESQUE, Michel. L'indexation: luxe ou necessite? Archives, Quebec, v. 33, n. 1, 2001, p. 17-45, 2001-2002.

LOPES, Luiz Carlos. A nova Arquivística na modernização administrativa. 2. ed. [S.1.: s.n.], 2009.

LYTLE, Richard H. Intellectual access to archives: II. report of an experiment comparing provenance and content indexing methods of subject retrieval. American Archivist, Menasha, v. 43, n. 2, p. 191-207, Spring 1980 . 
MACNEIL, Heather. Subject access to archival fonds: balancing provenance and pertinence. Fontes Artis Musicae, Kassel, v. 43, n. 3, p. 242-258, July/Sept.1996.

MCNITT, William H. Development of the PRESNET subject descriptor thesaurus. American Archivist, Menasha, v. 52, n. 3, p. 358-364, 1989.

OSTROFF, Harriet. Subject access to archival and manuscript material. American Archivist, Menasha, v. 53, n. 1, p. 100-105, 1990.

PINHEIRO, Lena Vânia Ribeiro. Campo interdisciplinar da Ciência da Informação: fronteiras remotas e recentes. Investigación Bibliotecológica, Ciudad de México, v. 12, n. 25, p.132-163, 1998.

PINHEIRO, Lena Vânia Ribeiro. Evolução e tendências da Ciência da Informação, no exterior e Brasil: quadro comparativo a partir de pesquisas históricas e empíricas. In: ORRICO, Evelyn Goyannes Dill. (Org.). ENCONTRO NACIONAL DE PESQUISA EM CIÊNCIA DA INFORMAÇÃO, 6., 2005, Florianópolis. Anais... Florianópolis: ANCIB, 2005.

RABELLO, Rodrigo. A Ciência da Informação como objeto: epistemologias como lugares de encontro. Perspectivas em Ciência da Informação, Belo Horizonte, v. 17, n. 1, p. 2-36, jan./mar. 2012.

RIBEIRO, Fernanda. Indexação e controlo de autoridade em arquivos. Porto: Câmara Municipal do Porto, 1996a. Disponível em: <http://repositorioaberto.up.pt/handle/10216/10721>. Acesso em: 18 fev. 2014.

RIBEIRO, Fernanda. O uso da classificação nos arquivos como instrumento de organização, representação e recuperação da informação. In: CONGRESSO ISKO ESPANHA E PORTUGAL, 1.; CONGRESO ISKO ESPAÑA 11., 2013, Porto. Atas... Porto: CETAC.MEDIA, 2013. Disponível em: < http://repositorioaberto.up.pt/bitstream/10216/69659/2/fernandaribeirousoclassificacao00021200 2.pdf>. Acesso em: 18 fev. 2014.

RIBEIRO, Fernanda. Subject indexing and authority control in archives: the need for subject indexing in archives and for an indexing policy using controlled language. Journal of the Society of Archivists, [S.1.], v. 17, n. 1, April, 1996b.

RODRIGUES, Georget Medleg. A representação da informação em arquivística: uma abordagem a partir da perspectiva da norma internacional de descrição arquivística. In: RODRIGUES, Georget Medleg; LOPES, Ilza Leite (Org.). Organização e representação do conhecimento na perspectiva da ciência da informação. Brasília: Thesaurus, 2003. p. 210-230. 
ROUSSEAU, Jean-Yves; COUTURE, Carol. Os fundamentos da disciplina arquivística. Lisboa: Dom Quixote, 1998. (Nova Enciclopédia, 56).

SARACEVIC, T. Ciência da Informação: origem, evolução e relações.

Perspectivas em Ciência da Informação, Belo Horizonte, v. 1, n. 1, p. 41-62, jan./jun. 1996.

SCHENKOLEWSKI-KROLL, Silvia; TRACTINSKY, Assaf. Archival description, information retrieval, and the construction of thesauri in Israeli archives. Archival Science, v. 6, n. 1, p. 69-107, 2006.

SCHMIDT, Clarissa. Entre o documento de arquivo e a informação arquivística: reflexões acerca do objeto científico da arquivologia. In: ENCONTRO NACIONAL DE PESQUISA EM CIÊNCIA DA INFORMAÇÃO 14., 2013, Florianópolis. Anais... Florianópolis: ANCIB, 2013. Disponível em: $<$ http://enancib.sites.ufsc.br/index.php/enancib2013/XIVenancib/paper/viewFile /408/242>. Acesso em: 18 fev. 2014.

SHERA, Jesse H.; CLEVELAND, Donald B. History and foundations of Information Science. Annual Review of Information Science and Technology, White Plains, v. 12, p. 249-275, 1977.

SILVA, Jonathas Luiza de Carvalho. Das concepções disciplinares na Ciência da Informação e/ou de suas configurações epistemológicas: o desiderato percepcionado da interdisciplinaridade. Investigación Bibliotecológica, Ciudad de México, v. 27, n. 59, 2013.

SMIRAGLIA, Richard P. Subject access to archival materials using LCSH. Cataloging and Classification Quarterly, New York, v. 11, n. 3-4, 1990.

SMIT, Johanna W. Recuperação, acesso e uso dos documentos arquivísticos. Ciência da Informação, Brasília, v. 41, n. 1, p. 11-23, jan./abr. 2013.

STOREY, Richard A. Indexing archives. Indexer, London, v. 5, n. 4, p.159168, Autumn 1967.

SOUSA, Renato Tarciso Barbosa de. A classificação como função matricial do que-fazer arquivistico. In: SANTOS, Vanderlei Batista dos; INNARELLI, Humberto Celeste (Org). Arquivistíca: temas contemporâneos: classificação, preservação digital, gestão do conhecimento 2. ed. Brasília: SENAC, 2008. p. 77-172. 


\title{
Subject indexing in archival documents: convergences and divergences in international articles
}

\begin{abstract}
This article aims to analyze the convergences and divergences presented in international scientific articles that deal with the subject indexing in archival documents, to advance the topic in the field of Archival and Information Science. The research is exploratory, descriptive and qualitative. Articles were collected in two databases: Library and information Science Abstracts and Library, Information Science \& Technologycal Abstracts. After preliminary analysis, we selected 20 articles, covering the period from 1965 to 2013. The results show that the definitions for indexing in Archivology roughly resemble the indexing of understanding in the context of Library and Information Science with need to consider the specific context, archival principles in the subject representation process and the development of guidelines for vocabulary control.
\end{abstract}

Keywords: Subject Indexing. Information representation. Archival documents

Recebido em: 17/06/2016

Aceito em: 19/10/2016

\footnotetext{
${ }^{1}$ MAUREL, D.; CHAMPAGNE, M. La description et l'indexation. In: COUTURE, Carol et al. Les fonctions de l'archivistique contemporaine. Sainte-Foy: Presses de l'Université du Québec, 1999. p. 255-371.
} 